\title{
MicroRNA-204 inhibits proliferation, migration, invasion and epithelial-mesenchymal transition in osteosarcoma cells via targeting Sirtuin 1
}

\author{
YING SHI ${ }^{1}$, JIANJUN HUANG ${ }^{2}$, JUN ZHOU ${ }^{1}$, YING LIU $^{1}$, XIAODAN FU $^{1}$, \\ YIMIN LI ${ }^{1}$, GANG YIN ${ }^{1}$ and JIFANG WEN ${ }^{1}$ \\ ${ }^{1}$ Department of Pathology, School of Basic Medicine, Central South University, Changsha, Hunan 410013; \\ ${ }^{2}$ The Second Department of Orthopedics, The First Affiliated Hospital of Jishou University, Jishou, Hunan 416000, P.R. China
}

Received December 8, 2014; Accepted March 5, 2015

DOI: 10.3892/or.2015.3986

\begin{abstract}
MicroRNAs (miRs) play crucial roles in tumorigenesis by directly suppressing the protein expression levels of their target genes. miR-204 has been suggested to act as a tumor suppressor in several types of human cancer. However, the exact role of miR-204 in osteosarcoma (OS) remains undetermined. In the present study, we aimed to investigate the effects of miR-204 on OS cell proliferation, migration and invasion, as well as the underlying molecular mechanisms. We found that the expression of miR-204 was frequently downregulated in four OS cell lines compared to the level in normal human osteoblast cells. Moreover, overexpression of miR-204 significantly inhibited the proliferation, migration and invasion of OS cells. Based on bioinformatics prediction and a luciferase reporter assay, we identified Sirtuin 1 (Sirt1) as a direct target gene of miR-204 in OS Saso-2 cells. Moreover, the protein expression of Sirt1 was negatively mediated by miR-204 in the OS cells. siRNA-mediated knockdown of Sirt1 also inhibited the proliferation, migration and invasion of the OS cells. Moreover, overexpression of Sirt1 reversed the inhibitory effect of miR-204 overexpression on the proliferation, migration and invasion of the OS cells. In addition, after miR-204 overexpression or Sirt1 knockdown in OS cells, the expression of E-cadherin was increased, while the $\mathrm{N}$-cadherin protein level was reduced. Based on these findings, we suggest that miR-204 inhibits the proliferation, migration, invasion and epithelial-mesenchymal transition (EMT) of OS cells by directly targeting Sirt1.
\end{abstract}

Correspondence to: Professor Jifang Wen, Department of Pathology, School of Basic Medicine, Central South University, 172 Tongzipo Road, Changsha, Hunan 410013, P.R. China

E-mail: csuwenjifang@163.com

Key words: osteosarcoma, microRNA-204, Sirtuin 1, proliferation, migration, invasion, epithelial-mesenchymal transition

\section{Introduction}

Osteosarcoma (OS), the most common mesenchymal sarcoma in bone, mainly arises from the metaphysis of the long bones of adolescents and young adults (1). Despite the fact that great efforts in the early diagnosis and effective treatment of OS have been achieved, the 5-year survival rate of patients with recurrent or metastatic OS remains $\sim 30 \%$ (1). As the deregulation of oncogenes or tumor suppressors has been found to play key roles in the growth and metastasis of OS, development of potential molecular targets shows promise for the effective therapy of OS (2).

MicroRNAs (miRs), a type of short non-coding RNAs, generally cause mRNA degradation or inhibition of protein translation, by directly binding to the 3'-untranslational region (3'-UTR) of their target mRNAs (3). By mediating the expression of their target genes, miRs play important roles in the regulation of various biological processes, including cell proliferation, differentiation, apoptosis, cell cycle progression and migration (4). Furthermore, both the upregulation of oncogenic miRs and the downregulation of tumor-suppressor miRs are involved in tumorigenesis and cancer metastasis $(5,6)$. Among these miRs, miR-204 generally acts as a tumor suppressor in human cancer. Li et al showed that downregulation of miR-204 was associated with poor prognosis in patients with breast cancer (7). Xia et al reported that miR-204 is downregulated in non-small cell lung cancer (NSCLC), and was found to inhibit proliferation, invasion and epithelialmesenchymal transition (EMT) by targeting SIX1 in NSCLC cells (8). However, the exact role of miR-204 in OS remains to be elucidated.

The present study aimed to reveal the exact role of miR-204 in the regulation of cell proliferation, migration, invasion and EMT in OS in vitro. In addition, we also investigated the underlying mechanisms, focusing on the target gene of miR-204 in OS cells.

\section{Materials and methods}

Cell culture. OS cell lines Saos-2, U-2 OS, HOS and MG-63 and the normal osteoblast cell line NHOst were obtained from 
the American Type Culture Collection (ATCC; Rockville, MD, USA). Cells were cultured in Dulbecco's modified Eagle's medium (DMEM) with 10\% fetal bovine serum (FBS) (both from Life Technologies, Carlsbad, CA, USA) at $37^{\circ} \mathrm{C}$ in a humidified incubator containing $5 \% \mathrm{CO}_{2}$.

Real-time RT-PCR assay. Total RNA was extracted using the miRNA Isolation kit (Life Technologies) according to the manufacturer's instructions. For detection of miR expression, the MicroRNA reverse transcription kit (Life Technologies) was used to convert $10 \mathrm{ng}$ of total RNA into cDNA, according to the manufacturer's instructions. Real-time PCR was then performed using the miRNA Q-PCR detection kit (GeneCopoeia, Rockville, MD, USA) on Applied Biosystems 7500 Real-Time PCR System. U6 gene was used as an internal reference. The relative miR-204 expression was normalized to U6. The relative expression was analyzed by the $2^{-\Delta \Delta \mathrm{Ct}}$ method.

Western blotting. Cells were lysed with ice-cold lysis buffer (50 mM Tris-HCl, pH 6.8, 100 mM 2-ME, 2\% w/v SDS, $10 \%$ glycerol). After centrifugation at $20,000 \mathrm{x}$ g for $10 \mathrm{~min}$ at $4{ }^{\circ} \mathrm{C}$, proteins in the supernatants were quantified and separated with 10\% SDS-PAGE. Then, proteins were transferred onto a polyvinylidene difluoride (PVDF) membrane (Amersham Biosciences, Buckinghamshire, UK), which was then incubated with phosphate-buffered saline (PBS) containing 5\% milk overnight at $4^{\circ} \mathrm{C}$. The PVDF membrane was incubated with rabbit anti-Sirt1 monoclonal antibody (1:100) and rabbit anti-GAPDH monoclonal antibody (1:200) at room temperature for $3 \mathrm{~h}$, respectively, and then with HRP-linked goat anti-rabbit secondary antibody (all from Abcam, Cambridge, UK) at room temperature for $1 \mathrm{~h}$. The Thermo Scientific SuperSignal West Pico Chemiluminescent Substrate kit (Pierce, Rockford, IL, USA) was then used to detect the signals according to the manufacturer's instructions. The relative protein expression was analyzed by Image-Pro Plus software 6.0, represented as the density ratio vs. GAPDH.

Transfection. Lipofectamine 2000 (Life Technologies) was used to perform cell transfection following the manufacturer's instructions. For functional analysis, Saos-2 and U-2 OS cells were transfected with scramble miR, miR-204 mimics, Sirt1 siRNA (all from Life Technologies) or co-transfected with miR-204 mimics and the Sirt1 plasmid (Nlunbio, Changsha, China), respectively.

Bioinformatics prediction. We screened the target genes of miR-204 using TargetScan (http://www.targetscan.org/index. html), PicTar (http://pictar.bio.nyu.edu/) and miRanda (http:// www.microrna.org/microrna/home.do).

Luciferase reporter assay. Luciferase reporter assay was performed to clarify whether Sirt1 is a direct target gene of miR-204. Briefly, total cDNA from OS Saos-2 cells was used to amplify the 3'-UTR of Sirt1, which was then cloned into the pMIR-REPORT vector (Life Technologies). Mutations were introduced within the potential seed sequences of the 3'-UTR of Sirt1 using the QuikChange Site-Directed Mutagenesis kit (Stratagene). The seed sequences AAAGGGAA were mutated into AAACCCAA. Using Lipofectamine 2000, the cells were transfected with the pMIR-REPORT vectors containing the wild-type or mutant type of Sirt1 3'-UTR and miR-204 mimics, respectively. The pRL-SV40 vector (Promega, USA) carrying the Renilla luciferase gene was used as an internal control. Luciferase activity was determined after $48 \mathrm{~h}$ using the Dual-Glo substrate system and LD400 luminometer (Beckman Coulter, Kraemer Boulevard, Brea, CA, USA). Data are presented as the ratio of Renilla luciferase to firefly luciferase.

Cell proliferation assay. The MTT assay was used to measure cell proliferation. At $48 \mathrm{~h}$ post-transfection, the transfection medium in each well was replaced with $100 \mu \mathrm{l}$ of fresh serum-free medium containing $0.5 \mathrm{~g} / 1$ MTT. Subsequent to incubation at $37^{\circ} \mathrm{C}$ for $4 \mathrm{~h}$, the MTT medium was removed by aspiration, and $50 \mu \mathrm{l}$ of dimethyl sulfoxide was added to each well. Following incubation at $37^{\circ} \mathrm{C}$ for a further $10 \mathrm{~min}$, the optical density at $570 \mathrm{~nm}$ was measured using the BioTek ${ }^{\mathrm{TM}}$ ELx800 ${ }^{\mathrm{TM}}$ Absorbance Microplate Reader (BioTek, Winooski, VT, USA)

Cell migration assay. A wound healing assay was performed to evaluate the cell migratory capacity of the OS cells in each group. In brief, OS cells were cultured to full confluency. Wounds of $\sim 1 \mathrm{~mm}$ in width were created with a plastic scriber, and cells were washed and incubated in a serum-free medium. After wounding for $24 \mathrm{~h}$, the cells were incubated in a medium including 10\% FBS. After being cultured for $48 \mathrm{~h}$, the cells were fixed and observed under a microscope.

Cell invasion assay. Cells in each group were starved in serum-free medium for $24 \mathrm{~h}$, and then resuspended in serumfree medium. The cell suspension was added into the upper chamber, while the lower chamber was filled with base medium containing 10\% FBS. After incubation for $24 \mathrm{~h}$, the cells that attached to the bottom were stained with crystal violet for $20 \mathrm{~min}$, and then washed and dried in air. Invasive cells were observed under a microscope.

Statistical analysis. Data are expressed as mean \pm standard deviation from at least three separate experiments. Differences were analyzed using one-way analysis of variance (ANOVA). SPSS 18.0 software was used to perform statistical analysis. $\mathrm{P}<0.05$ was considered to indicate a statistically significant result.

\section{Results}

miR-204 is downregulated in the OS cells. Real-time RT-PCR was used to detect the expression of miR-204 in four OS cell lines Saos-2, U-2 OS, HOS and MG-63, and in the normal osteoblast cell line NHOst. Our data showed that the expression of miR-204 was significantly reduced in the OS cell lines, when compared with that in the normal osteoblast cell line NHOst (Fig. 1). We then chose Saos-2 and U-2 OS cells to perform functional analysis of miR-204 in OS in vitro.

Upregulation of miR-204 inhibits the proliferation, migration and invasion of OS cells. The role of miR-204 in the regulation of proliferation, migration and invasion of OS cells was 


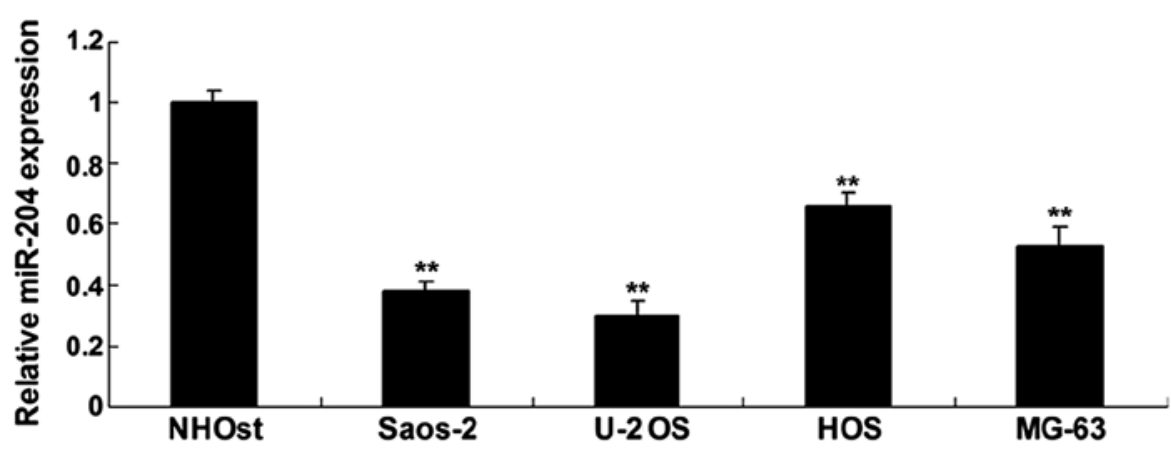

Figure 1. Real-time RT-PCR was performed to examine the relative expression of miR-204 in four OS cell lines, Saos-2, U-2 OS, HOS and MG-63, and the normal osteoblast cell line NHOst. ${ }^{* *} \mathrm{P}<0.01$ vs. NHOst.
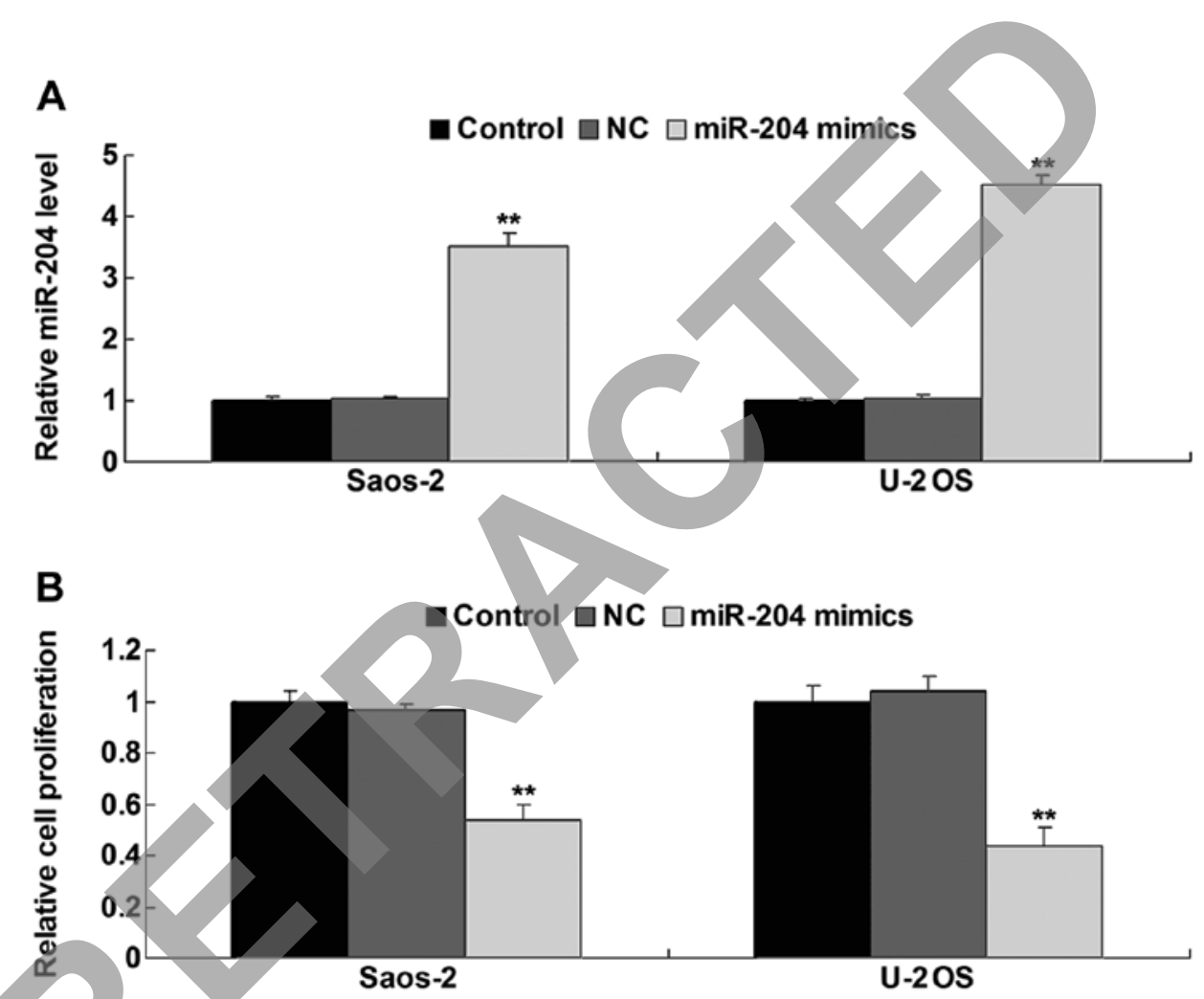

Figure 2. (A) Real-time RT-PCR was performed to examine the relative expression of miR-204 in Saos-2 and U-2 OS cells transfected with negative control (NC) scramble miRNA or miR-204 mimics, respectively. Control, Saos-2 and U-2 OS cells without any transfection. ${ }^{* *} \mathrm{P}<0.01$ vs. control. (B) Cell proliferation assay was performed in Saos-2 and U-2 OS cells transfected with NC scramble miRNA or miR-204 mimics, respectively. Control, Saos-2 and U-2 OS cells without any transfection. ${ }^{* *} \mathrm{P}<0.01$ vs. control.

further investigated. After transfection of the Saos-2 and U-2 OS cells with miR-204 mimics, we firstly determined the level of miR-204 in each group. As shown in Fig. 2A, the miR-204 level was significantly increased after transfection compared to the control group. Cell proliferation assay data showed that overexpression of miR-204 notably inhibited the proliferation of the Saos-2 and U-2 OS cells, when compared to the control groups (Fig. 2B). Subsequently, we determined the cell migration by performing a scratch assay. As shown in Fig. 3A, the migratory capacity of the OS cells transfected with the miR-204 mimics was significantly reduced, when compared to this capacity in the control groups. Moreover, miR-204 overexpression also suppressed the invasive capacity of the Saos-2 and U-2 OS cells, when compared to the control groups (Fig. 3B). These findings suggest that miR-204 plays an inhibitory role in the regulation of proliferation, migration and invasion of OS cells.

Sirtl is identified as a target gene of $m i R-204$. According to bioinformatics predication, Sirt1 is a putative target gene of miR-204 (Fig. 4A). To confirm that Sirt1 is a target gene of miR-204, the luciferase reporter assay was performed to clarify whether miR-204 directly binds to their seed sequences in the Sirtl 3'-UTR in the Saos-2 cells. The luciferase activity was significantly decreased in the Saos-2 cells co-transfected with the wild-type (WT) Sirt1 3'-UTR and miR-204 mimics, but showed no difference in the Saos-2 cells co-transfected with the mutant type (MUT) Sirt1 3'-UTR and miR-204 mimics, when compared with that in the control groups (Fig. 4B). These data indicate that Sirt1 is a target gene of miR-204. We then 
A

Saos-2 NC
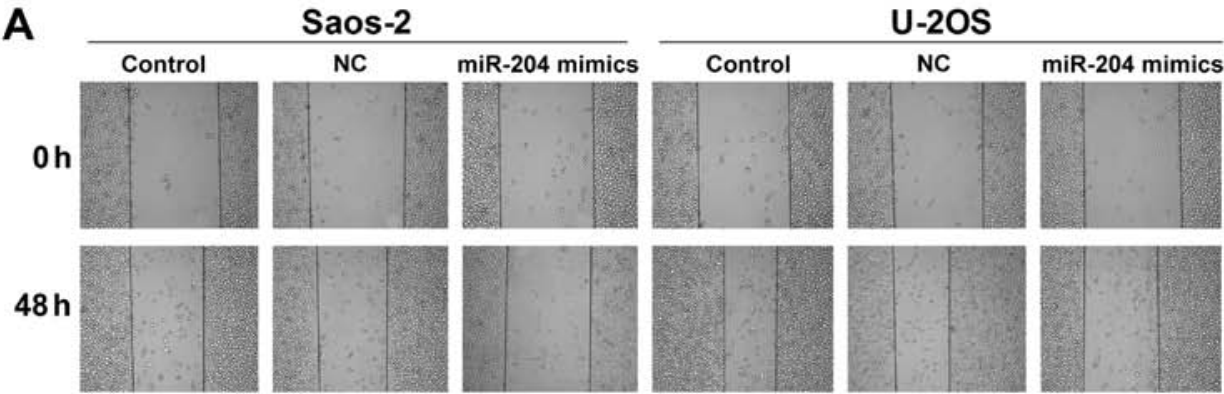

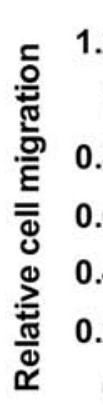

$\square$ Control $\square$ NC $\square$ miR-204 mimics
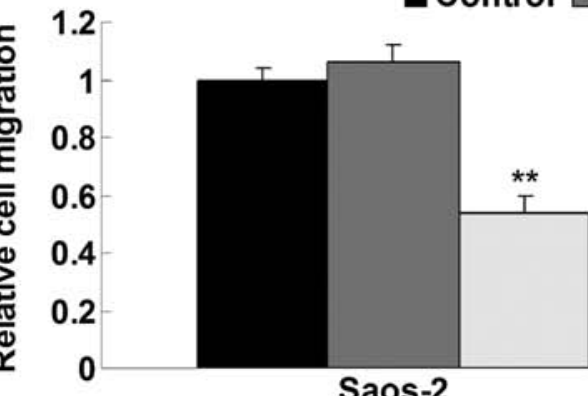

Saos-2
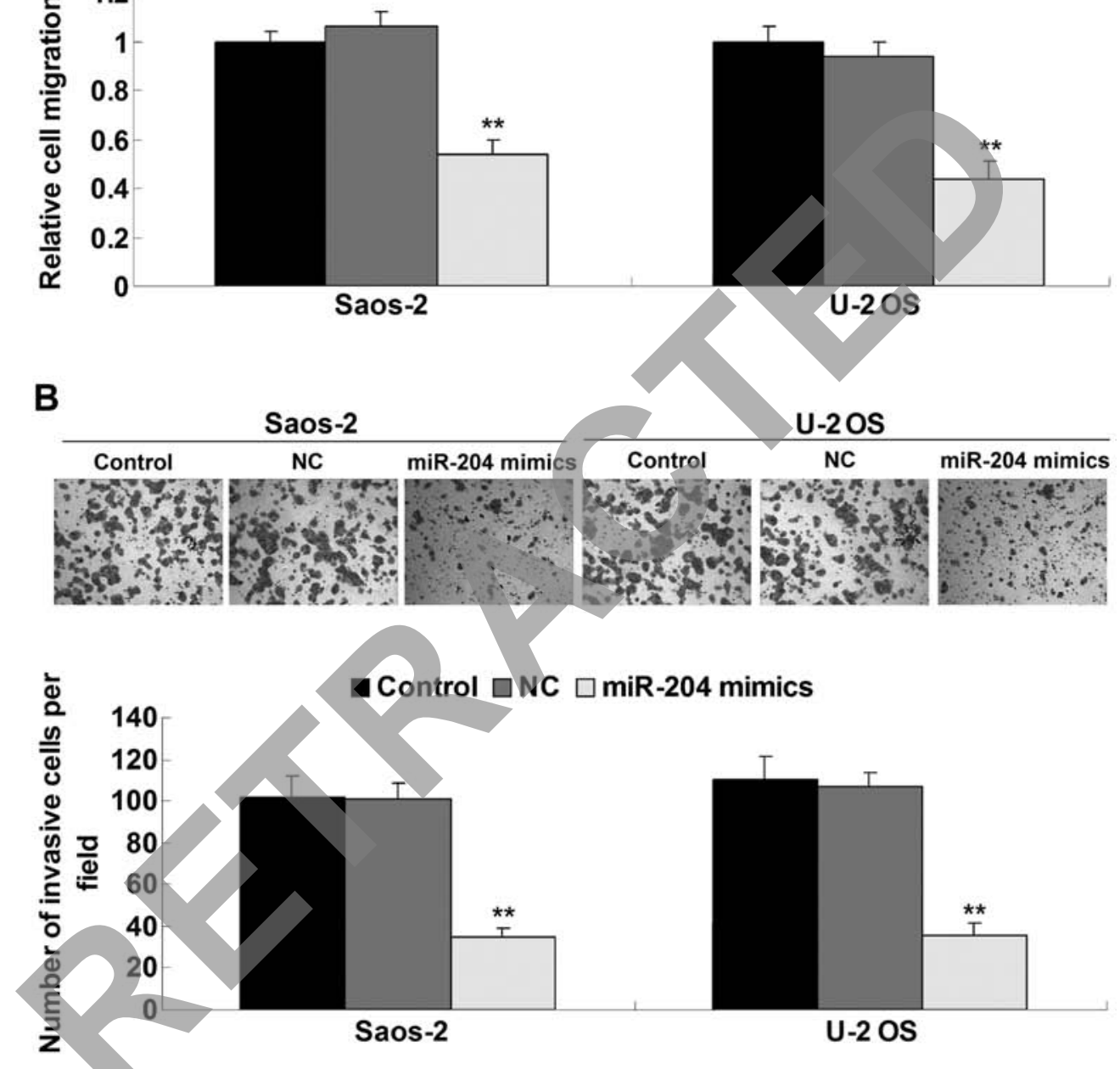

Control $\square$ NC $\square$ miR-204 mimics

Figure 3. (A) Scratch assay was performed to determine the migratory capacity of Saos-2 and U-2 OS cells transfected with negative control (NC) scramble miRNA or miR-204 mimics, respectively. Control, Saos-2 and U-2 OS cells without any transfection. ${ }^{* *} \mathrm{P}<0.01$ vs. control. (B) Transwell assay was performed to determine the invasive capacity of the Saos-2 and U-2 OS cells transfected with NC scramble miRNA or miR-204 mimics, respectively. Control, Saos-2 and $\mathrm{U}-2$ OS cells without any transfection. ${ }^{* *} \mathrm{P}<0.01$ vs. control.

detected the protein level of Sirt1 in the Saos-2 and U-2 OS cells with or without transfection with the miR-204 mimics. As shown in Fig. 4C, overexpression of miR-204 inhibited the protein level of Sirt1 in the OS cells when compared to the control groups, indicating that miR-204 negatively mediates the protein expression of Sirt1 through directly binding to the 3'-UTR in Sirt1 mRNA.

Sirtl is involved in miR-204-mediated proliferation, migration and invasion of OS cells. To further clarify whether Sirt1 acts as a downstream effector in miR-204-mediated proliferation, migration and invasion of OS cells, we transfected
Saos-2 and U-2 OS cells with Sirtl siRNA or co-transfected the cells with miR-204 mimics and the Sirt1 plasmid. After that, we firstly determined the protein expression of Sirt1 by performing western blotting in each group. As shown in Fig. 5A, transfection with Sirt1 siRNA significantly inhibited the protein expression of Sirt1 in the OS cells when compared to that in the control groups, while transfection with the Sirt1 plasmid reversed the inhibitory effect of miR-204 overexpression on Sirt1 protein expression in the OS cells. Subsequently, we determined the proliferation, migratory and invasive capacities of the OS cells in each group. Our data showed that inhibition of Sirt1 significantly inhibited OS cell prolif- 


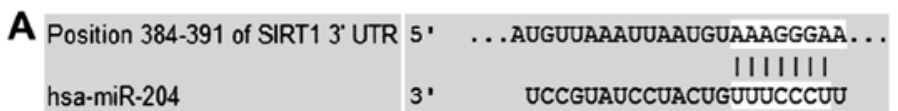

B

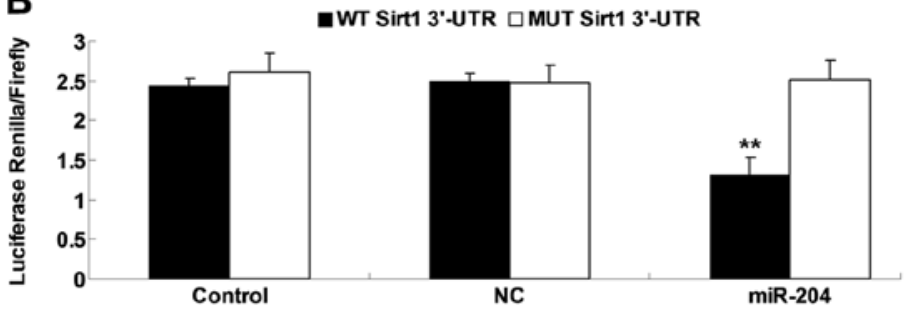

C
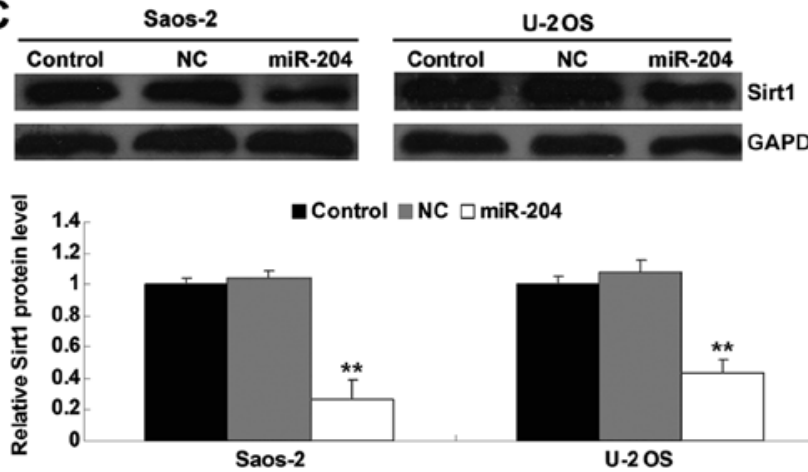

Figure 4. (A) The seed sequences of miR-204 in the 3'-UTR of Sirt1 were indicated by Targetscan software. (B) Luciferase reporter assay data found that co-transfection of Saos-2 cells with miR-204 and wild-type (WT) Sirt1 3'-UTR caused a significant decrease in luciferase activity, whereas co-transfection with mutant-type (MUT) Sirt1 3'-UTR and miR-204 mimics showed no difference with the control group. Control, cells co-transfected with blank vector and WT Sirt1 3'-UTR or MUT Sirt1 3'-UTR. ${ }^{* *} \mathrm{P}<0.01$ vs. control. Western blot assay was performed to examine the protein level of Sirt1 in the Saos-2 and U-2 OS cells transfected with negative control (NC) scramble miRNA or miR-204 mimics, respectively. GAPDH was used as an internal reference. Control, Saos-2 and U-2 OS cells without any transfection. ${ }^{* *} \mathrm{P}<0.01$ vs. control. Sirt1, Sirtuin 1.

A

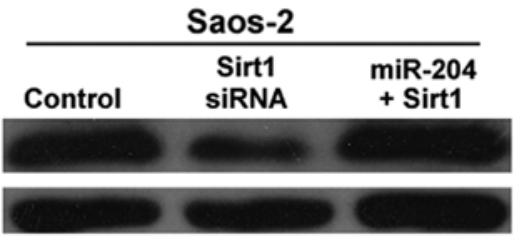

U-2 OS
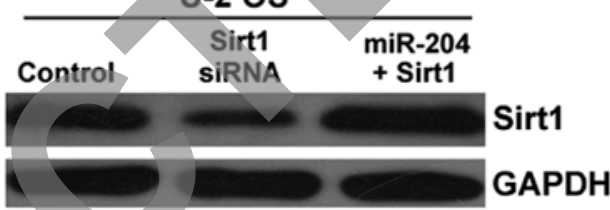

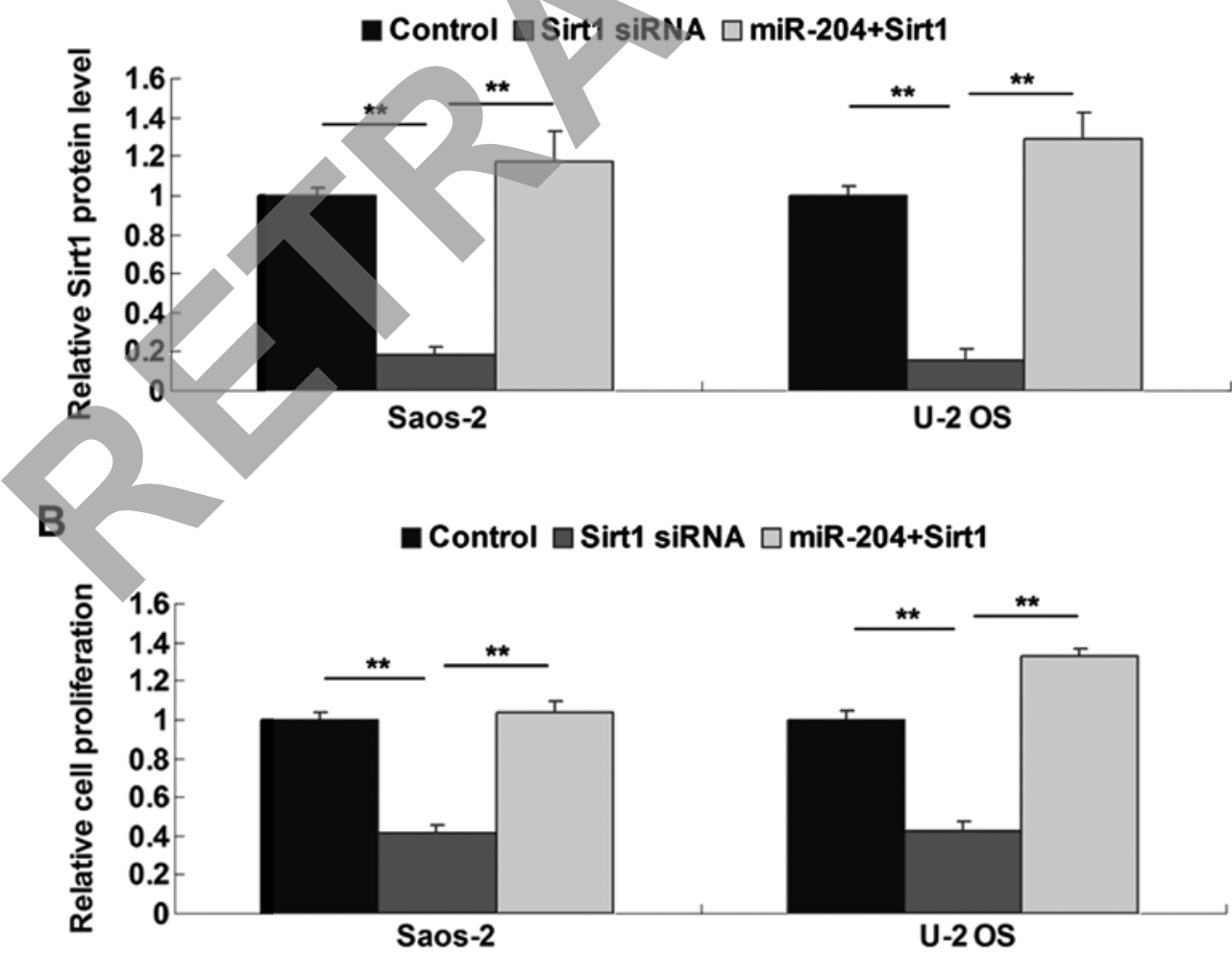

Figure 5. (A) Western blot assay was performed to determine the protein level of Sirt1 in the Saos-2 and U-2 OS cells transfected with Sirt1 siRNA or co-transfected with miR-204 mimics and the Sirt1 plasmid, respectively. GAPDH was used as an internal reference. Control, Saos-2 and U-2 OS cells without any transfection. ${ }^{* *} \mathrm{P}<0.01$. (B) Cell proliferation assay was performed in Saos-2 and U-2 OS cells transfected with Sirt1 siRNA, or co-transfected with miR-204 mimics and the Sirt1 plasmid, respectively. Control, Saos-2 and U-2 OS cells without any transfection. ${ }^{* *} \mathrm{P}<0.01$. Sirt1, Sirtuin 1.

eration (Fig. 5B), migration (Fig. 6A) and invasion (Fig. 6B). However, overexpression of Sirt1 reversed the inhibitory effect of miR-204 upregulation on OS cell proliferation, migration and invasion (Figs. 5B, and 6A and B), suggesting that Sirt1 


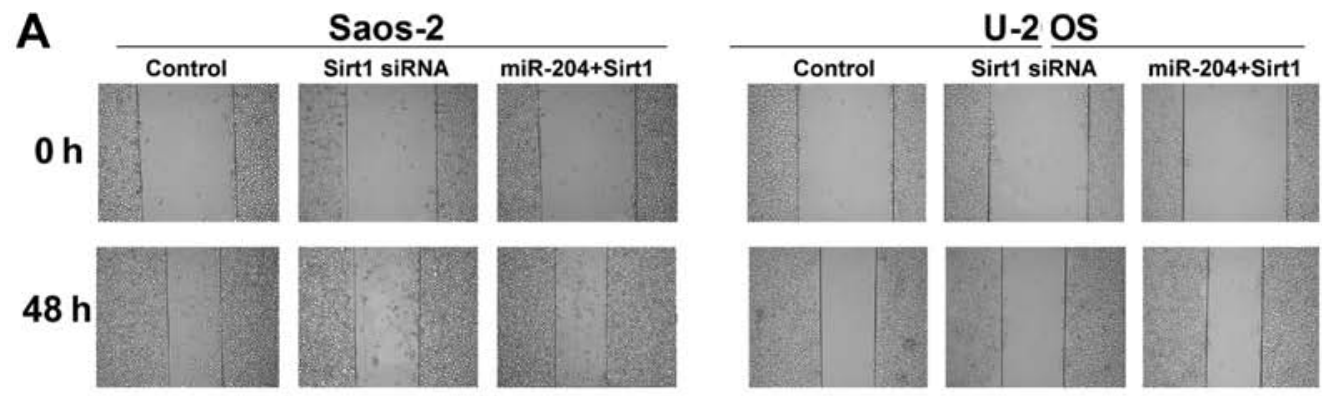

-Control $\square$ Sirt1 siRNA $\square$ miR-204+Sirt1
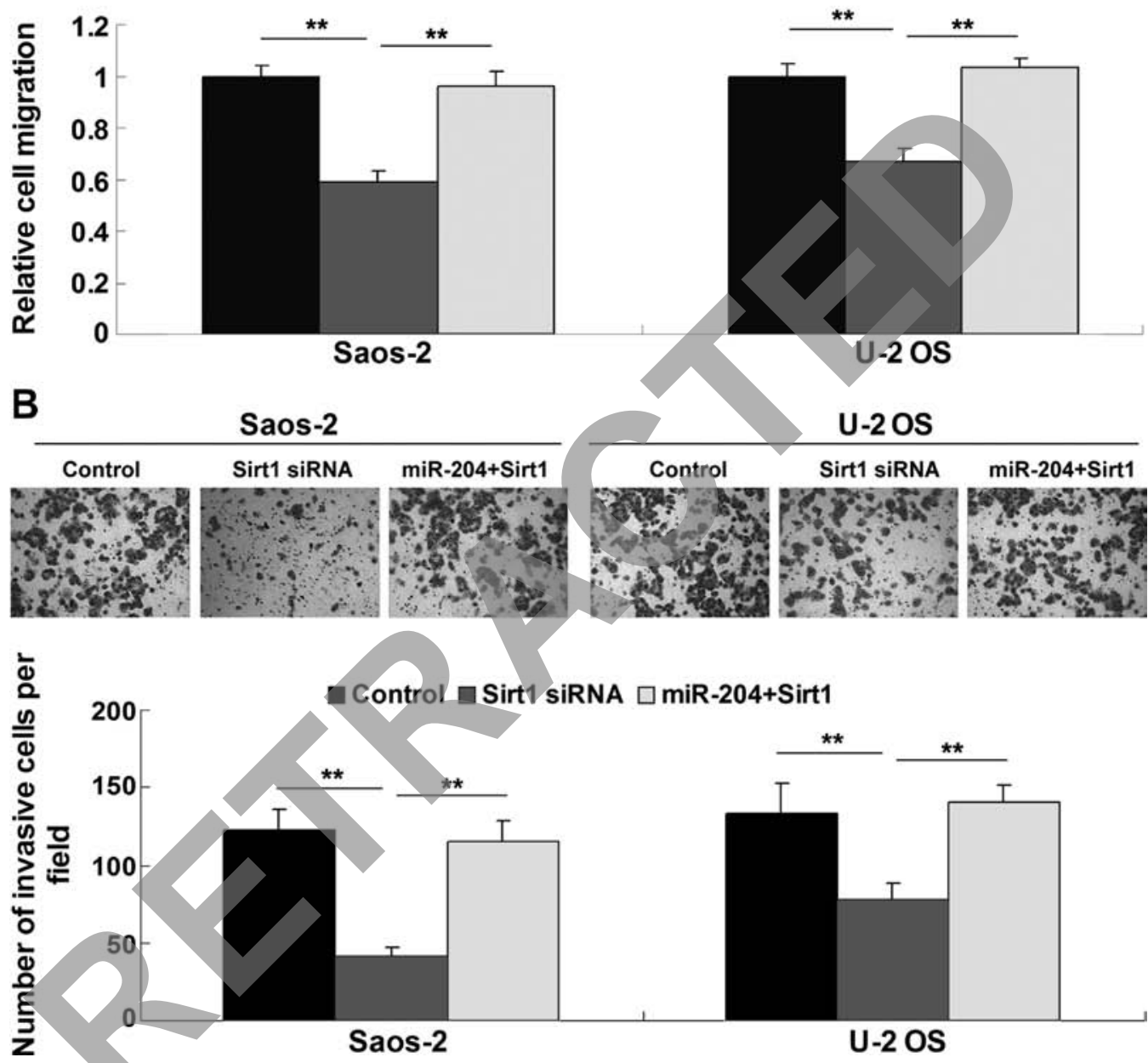

Figure 6. (A) Scratch assay was performed to determine the migratory capacity of the Saos-2 and U-2 OS cells transfected with Sirt1 siRNA, or co-transfected with miR-204 mimics and the Sirt1 plasmid, respectively. Control, Saos-2 and U-2 OS cells without any transfection. ${ }^{* *} \mathrm{P}<0.01$. (B) Transwell assay was performed to determine the invasive capacity of the Saos-2 and U-2 OS cells transfected with Sirt1 siRNA or co-transfected with miR-204 mimics and the Sirt1 plasmid, respectively. Control, Saos-2 and U-2 OS cells without any transfection. ${ }^{* *} \mathrm{P}<0.01$. Sirt1, Sirtuin 1.

is involved in the miR-204-mediated proliferation, migration and invasion of OS cells.

miR-204 and Sirt1 are involved in EMT of OS cells. As EMT is also a key step in cancer metastasis in addition to migration and invasion, we further investigated the roles of miR-204 and Sirt1 in the EMT of OS cells. The protein expression of N-cadherin and E-cadherin was detected in the OS cells transfected with miR-204 mimics or Sirt1 siRNA using western blotting. As shown in Fig. 7, the protein expression of E-cadherin was increased, while the N-cadherin protein level was decreased after miR-204 overexpression or Sirt1 knockdown in the OS cells, suggesting that miR-204 plays a suppressive role of EMT in OS cells via targeting Sirtl.

\section{Discussion}

Tumor cell proliferation, migration, invasion and EMT play key roles in tumor growth and metastasis. miR-204 generally acts as a tumor suppressor in human types of cancer, yet its exact role in OS remains unclear. In the present study, we showed that miR-204 was downregulated in OS cells compared to normal osteoblast cells, and played a suppressive role in the regulation of proliferation, migration and invasion of OS cells. Further 
Control Sirt1 siRNA miR-204 mimics
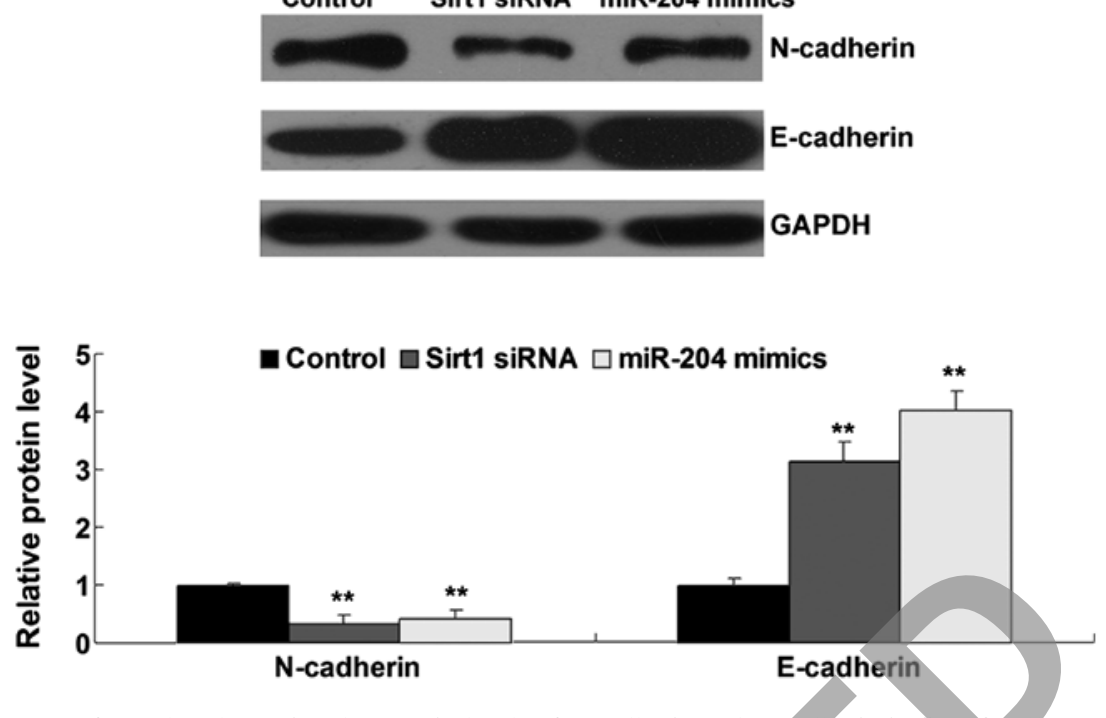

Figure 7. Western blot assay was performed to determine the protein levels of E-cadherin and N-cadherin in Saos-2 cells transfected with miR-204 mimics or Sirt1 siRNA, respectively. GAPDH was used as an internal reference. Control, Saos- 2 cells without any transfection. ${ }^{* *} \mathrm{P}<0.01$ vs. control. Sirt1, Sirtuin 1 .

investigation identified Sirt1 as a direct target of miR-204 in the OS cells, which was then found to be associated with miR-204-mediated OS cell proliferation, migration and invasion. In addition, we also found that miR-204 and Sirt1 play important roles in the regulation of EMT in OS cells.

It has been well established that deregulation of miRs is tightly associated with the development and progression of various types of human cancer including OS. For example, miR-451 expression is associated with the prognosis of OS patients, and it inhibits OS cell growth and invasion by targeting CXCL16 (9). miR-382 was found to inhibit tumor growth and enhance chemosensitivity in OS (10). He et al found that miR-23 plays an inhibitory role in OS (11). Other miRs were also reported to act as oncogenes or tumor suppressors in OS, such as miR-101, miR-126, miR-143, miR-194 and miR-217 (12-17). In the present study, we showed for the first time that miR-204 acted as a tumor suppressor in OS in vitro. Overexpression of miR-204 significantly inhibited OS cell proliferation, migration and invasion. Similar findings were also reported in other types of cancer. For example, Shi et al showed that decreased expression of miR-204 promoted NSCLC progression, while overexpression of miR-204 inhibited cell migration and invasion of NSCLC cells (18). Zhou et al found that knockdown of miR-204 enhanced the proliferation and invasion ability of gastric cancer cells in vitro (19). Moreover, Ying et al reported that overexpression of miR-204 expression suppressed tumorigenesis and invasiveness in glioma cells (20).

As miRs play roles in human cancer via mediating the protein expression of their target genes (21), we further focused on the target of miR-204 in OS cells. Our data indicated that Sirtl was a direct target gene of miR-204, and its protein level was negatively mediated by miR-204 in OS cells. In fact, this targeting relationship has also recently been demonstrated in gastric cancer cells (22). Sirt1, a type of class III histone deacetylase, has been found to promote cell growth and angiogenesis, and block senescence and apoptosis, thus playing a key role in tumorigenesis and metastasis $(23,24)$. In fact, the oncogenic role of Sirt1 has been previously suggested in OS.
Xu et al found that Sirt1 is a direct target of miR-126, which inhibited OS cell proliferation via inhibition of Sirt1 expression (25). Moreover, Sirt1 was also suggested to be associated with resistance to chemotherapy in OS cells $(26,27)$. In the present study, siRNA-mediated Sirt1 inhibition significantly suppressed proliferation, migration and invasion in OS cells, similar with the effects of miR-204 overexpression. Moreover, we found that overexpression of Sirt1 reversed the inhibitory effect of miR-204 upregulation on OS cell proliferation, migration and invasion, further confirming that miR-204 inhibited the malignant phenotypes of OS cells via targeting Sirt1.

EMT, a key step in cancer metastasis, in a process where epithelial cells lose epithelial characteristics and acquire mesenchymal characteristics (28). As a hallmark of EMT, loss of E-cadherin expression is likely required for enhanced tumor cell motility. Moreover, upregulation of $\mathrm{N}$-cadherin is another hallmark of EMT (29). In the present study, we found that both overexpression of miR-204 and knockdown of Sirt1 led to decreased expression of $\mathrm{N}$-cadherin and increased expression of E-cadherin in OS cells, suggesting that miR-204 inhibits EMT while Sirt1 promotes EMT in OS. As miR-204 negatively regulated the Sirt1 expression in OS cells, we suggest that the effect of miR-204 on EMT in OS cells may be through the modulation of its target Sirt1.

In addition, other miR-204 targets have also been identified, which act as oncogenes in various human types of cancer. For example, MAP1LC3B (LC3B) is a direct and functional target of miR-204, and miR-204 suppresses tumor growth through inhibition of LC3B-mediated autophagy in renal clear cell carcinoma (30). Sacconi et al showed that miR-204 targeted Bcl-2 messenger RNA and increased the responsiveness of gastric cancer cells to 5-fluorouracil and oxaliplatin treatment (31). Moreover, miR-204 induced pancreatic cancer cell death via targeting Mcl-1 (32). In addition, NUAK1, RAB22A and SOX4, which play oncogenic roles, were also identified as direct targets of miR-204 in human types of cancer $(18,19,33)$. Therefore, the present study expands the understanding of the targets of miR-204 in human cancer. 
In conclusion, miR-204 plays an inhibitory role in the regulation of cell proliferation, migration, invasion and EMT of OS cells via targeting Sirt1, highlighting the significance of miR-204 and Sirt1 in molecular-targeted therapy for OS.

\section{Acknowledgements}

The present study was supported by grants from the National Natural Science Foundation of China (no. 81201675), the Research Project of the Education Department of Hunan Province (11C1035), the Natural Science Fund Project of Hunan Province (12JJ6074), the Scientific Research Project of the Health Department of Hunan Province (B2013-160), and the Talent Introduction Research of Jishou University (jsdxrcyjkyxm201110).

\section{References}

1. Thompson LD: Osteosarcoma. Ear Nose Throat J 92: 288, 290, 2013.

2. Liang W, Gao B, Fu P, Xu S, Qian Y and Fu Q: The miRNAs in the pathgenesis of osteosarcoma. Front Biosci 18: 788-794, 2013.

3. Moss EG: MicroRNAs: Hidden in the genome. Curr Biol 12: R138-R140, 2002.

4. Choi E, Choi E and Hwang KC: MicroRNAs as novel regulators of stem cell fate. World J Stem Cells 5: 172-187, 2013.

5. Lujambio A, Calin GA, Villanueva A, Ropero S, SánchezCéspedes M, Blanco D, Montuenga LM, Rossi S, Nicoloso MS, Faller WJ, et al: A microRNA DNA methylation signature for human cancer metastasis. Proc Natl Acad Sci USA 105 13556-13561, 2008

6. Calin GA and Croce CM: MicroRNA signatures in human cancers. Nat Rev Cancer 6: 857-866, 2006.

7. Li W, Jin X, Zhang Q, Zhang G, Deng X and Ma L: Decreased expression of miR-204 is associated with poor prognosis in patients with breast cancer. Int J Clin Exp Pathol 7. 3287-3292, 2014.

8. Xia Y, Zhu Y, Ma T, Pan C, Wang J, He Z,Li Z, Qi X and Chen Y: miR-204 functions as a tumor suppressor by regulating SIX1 in NSCLC. FEBS Lett 588: 3703-3712, 2014.

9. Zhang F, Huang W, Sheng M and Liu T: MiR-451 inhibits cell growth and invasion by targeting CXCL16 and is associated with prognosis of osteosarcoma patients. Tumour Biol: Nov 13, 2014 (Epub ahead of print).

10. Xu M, Jin H, Xu CX, Sun B, Mao Z, Bi WZ and Wang Y: miR-382 inhibits tumor growth and enhance chemosensitivity in osteosarcoma. Oncotarget 5: 9472-9483, 2014.

11. He Y, Meng C, Shao Z, Wang H and Yang S: MiR-23a functions as a tumor suppressor in osteosarcoma. Cell Physiol Biochem 34 1485-1496, 2014.

12. Shen L, Wang P, Yang J and Li X: MicroRNA-217 regulates WASF3 expression and suppresses tumor growth and metastasis in osteosarcoma. PLoS One 9: e109138, 2014.

13. Wang Q, Cai J, Wang J, Xiong C and Zhao J: MiR-143 inhibits EGFR-signaling-dependent osteosarcoma invasion. Tumour Biol 35: 12743-12748, 2014

14. Jiang L, He A, Zhang Q and Tao C: miR-126 inhibits cell growth, invasion, and migration of osteosarcoma cells by downregulating ADAM-9. Tumour Biol 35: 12645-12654, 2014.

15. Zhang K, Zhang Y, Ren K, Zhao G, Yan K and Ma B: MicroRNA-101 inhibits the metastasis of osteosarcoma cells by downregulation of EZH2 expression. Oncol Rep 32: 2143-2149, 2014.
16. Han K, Zhao T, Chen X, Bian N, Yang T, Ma Q, Cai C, Fan Q, Zhou Y and Ma B: microRNA-194 suppresses osteosarcoma cell proliferation and metastasis in vitro and in vivo by targeting CDH2 and IGF1R. Int J Oncol 45: 1437-1449, 2014.

17. Yang J and Zhang W: New molecular insights into osteosarcoma targeted therapy. Curr Opin Oncol 25: 398-406, 2013

18. Shi L, Zhang B, Sun X, Lu S, Liu Z, Liu Y, Li H, Wang L, Wang X and Zhao C: MiR-204 inhibits human NSCLC metastasis through suppression of NUAK1. Br J Cancer 111: 2316-2327, 2014

19. Zhou X, Li L, Su J and Zhang G: Decreased miR-204 in H. pyloriassociated gastric cancer promotes cancer cell proliferation and invasion by targeting SOX4. PLoS One 9: e101457, 2014.

20. Ying Z, Li Y, Wu J, Zhu X, Yang Y, Tian H, Li W, Hu B, Cheng SY and Li M: Loss of miR-204 expression enhances glioma migration and stem cell-like phenotype. Cancer Res 73: 990-999, 2013.

21. Yoshitaka T, Kawai A, Miyaki S, Numoto K, Kikuta K, Ozaki T, Lotz $\mathrm{M}$ and Asahara $\mathrm{H}$ : Analysis of microRNAs expressions in chondrosarcoma. J Orthop Res 31: 1992-1998, 2013.

22. Zhang L, Wang X and Chen P: MiR-204 down regulates SIRT1 and reverts SIRT1-induced epithelial-mesenchymal transition, anoikis resistance and invasion in gastric cancer cells. BMC Cancer 13: 290, 2013.

23. Li D, Bi FF, Chen NN, Cao JM, Sun WP, Zhou YM, Li CY and Yang Q: A novel crosstalk between BRCA1 and sirtuin 1 in ovarian cancer. Sci Rep 4: 6666, 2014.

24. Lin L, Zheng X, Qiu C, Dongol S, Lv Q, Jiang J, Kong B and Wang C: SIRT1 promotes endometrial tumor growth by targeting SREBP1 and lipogenesis. Oncol Rep 32: 2831-2835, 2014.

25. Xu JQ, Liu P, Si MJ and Ding XY: MicroRNA-126 inhibits osteosarcoma cells proliferation by targeting Sirt1. Tumour Biol 34: 3871-3877, 2013.

26. Chu F, Chou PM, Zheng X, Mirkin BL and Rebbaa A: Control of multidrug resistance gene $m d r l$ and cancer resistance to chemotherapy by the longevity gene sirt1. Cancer Res 65: 10183-10187, 2005.

27. Li Y, Bäckesjö CM, Haldosén LA and Lindgren U: Resveratrol inhibits proliferation and promotes apoptosis of osteosarcoma cells. Eur J Pharmacol 609: 13-18, 2009.

28. Moustakas A and Heldin CH: Signaling networks guiding epithelial-mesenchymal transitions during embryogenesis and cancer progression. Cancer Sci 98: 1512-1520, 2007.

29. Thiery JP and Sleeman JP: Complex networks orchestrate epithelial-mesenchymal transitions. Nat Rev Mol Cell Biol 7: 131-142, 2006.

30. Mikhaylova O, Stratton Y, Hall D, Kellner E, Ehmer B, Drew AF, Gallo CA, Plas DR, Biesiada J, Meller J, et al: VHL-regulated miR-204 suppresses tumor growth through inhibition of LC3Bmediated autophagy in renal clear cell carcinoma. Cancer Cell 21: 532-546, 2012.

31. Sacconi A, Biagioni F, Canu V, Mori F, Di Benedetto A, Lorenzon L, Ercolani C, Di Agostino S, Cambria AM, Germoni S, et al: miR-204 targets Bcl-2 expression and enhances responsiveness of gastric cancer. Cell Death Dis 3: e423, 2012.

32. Chen Z, Sangwan V, Banerjee S, Mackenzie T, Dudeja V, Li X, Wang H, Vickers SM and Saluja AK: miR-204 mediated loss of Myeloid cell leukemia-1 results in pancreatic cancer cell death. Mol Cancer 12: 105, 2013.

33. Yin Y, Zhang B, Wang W, Fei B, Quan C, Zhang J, Song M, Bian Z, Wang Q, Ni S, et al: miR-204-5p inhibits proliferation and invasion and enhances chemotherapeutic sensitivity of colorectal cancer cells by downregulating RAB22A. Clin Cancer Res 20: 6187-6199, 2014. 